\title{
A REMARK ON ROBERTS' TOTALLY TWISTED KHOVANOV HOMOLOGY
}

\author{
THOMAS C. JAEGER
}

\begin{abstract}
We offer an alternative construction of Roberts' totally twisted Khovanov homology and prove that it agrees with $\delta$-graded reduced characteristic-2 Khovanov homology.
\end{abstract}

\section{INTRODUCTION}

Spanning tree models have been successfully used to study both knot Floer homology and Khovanov homology of (quasi-)alternating links. They appear to occur in two flavors: On the one hand, the models described by Ozsváth and Szabó for knot Floer homology [5] and by Wehrli [7] and Champanerkar and Kofman [4] for Khovanov homology completely determine the homology of the knot, but their differentials are in general difficult to compute. On the other hand, the spanning tree models recently introduced by Baldwin and Levine for knot Floer homology [1] and by Roberts for Khovanov homology [6] have completely explicit differentials, but only recover the homology with the bigrading collapsed to a single $\delta$-grading.

In the present note, we answer a question raised by Roberts, showing that for knots, his theory indeed coincides with $\delta$-graded reduced Khovanov homology.

\section{Definitions}

In order to be able to express the theory locally, we will need to relax the notion of a chain complex:

Definition 2.1. If $\mathcal{C}$ is a graded additive category whose Hom-sets are $\mathbb{F}_{2^{-}}$ algebras, then we say that a chain complex over $\mathcal{C}$ is a an object $C$ of $\mathcal{C}$ together with a differential $d: C \rightarrow C$ of degree 2 such that $d^{2}=0$.

When we deal with multiple gradings, we will refer to this grading as the homological grading. Based on this definition, it is straightforward to redefine basic notions of homological algebra: A chain morphism between two chain complexes is a degree-0 morphism between the underlying objects that commutes with the differentials and a chain homotopy between chain morphisms $f: C \rightarrow C^{\prime}$ and $g: C \rightarrow C^{\prime}$ is a morphism $h: C^{\prime} \rightarrow C$ of degree -2 such that $f+g=d h+h d$. Given a tensor product $\otimes: \mathcal{C}_{1} \times \mathcal{C}_{2} \rightarrow \mathcal{C}$, we define the tensor product of chain complexes $C_{1} \in \mathcal{C}_{1}$ and $C_{2} \in \mathcal{C}_{2}$ as the object $C=C_{1} \otimes C_{2}$ with differential $d=d_{1} \otimes 1+1 \otimes d_{2}$. It is easy to 
see that this operation is associative, but note that this uses the assumption that we work in characteristic 2 in an essential way. In the familiar case that the category $\mathcal{C}$ is the category of graded modules over an (ungraded) ring $R$, the above notions clearly agree with the usual definitions.

For our definition of Khovanov homology, we follow Bar-Natan's approach (see [2] for the general ideas and [3] for a modification that works in characteristic 2). Let $R$ be a ring of characteristic 2 . The Khovanov homology of a tangle with endpoints $E$ is a chain complex (in the above sense) over a certain bigraded category $\operatorname{Kob}(E)$ of crossingless tangles and $R$-linear combinations of dotted cobordisms subject to certain relations modeled after the Frobenius algebra structure of $R[x] /\left(x^{2}\right)$. Specifically, the complexes associated to the positive and negative crossings are

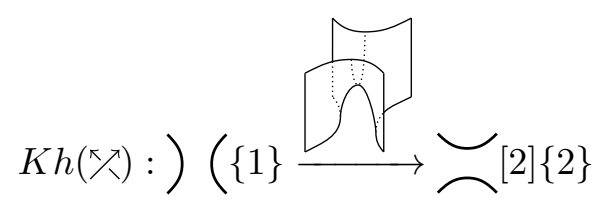

and

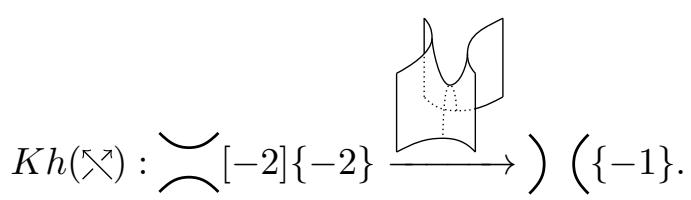

We write $)$ - for the differential of $K h(\kappa)$ and $\nearrow$ for the differential of $K h\left(\nwarrow^{\top}\right)$. Here [.] denotes a shift in homological grading and $\{\cdot\}$ a shift in $q$-grading (which is defined using the Euler characteristic of the cobordisms). We use the following conventions: The $q$-grading of a dot is -2 ; if $f: C \rightarrow C^{\prime}$ is a morphism of $q$-degree $d$, then the corresponding morphism $f: C\{n\} \rightarrow$ $C\{m\}$ has $q$-degree $d+m-n$ and similarly for homological degree.

The reduced Khovanov homology of a link $L$ can now be defined as follows: $K h(L)$ is computed by tensoring the Khovanov complexes of the individual crossings. $K h(L)$ is a complex of free $R[x]$-modules, where $x$ is the action of the dot at the basepoint. Here we used implicitly that $\mathbf{K o b}(\emptyset)$ is equivalent to the category of finitely generated free $R$-modules. The reduced Khovanov complex is simply $\overline{K h}(L):=K h(L) \otimes_{R[x]} R$, where $x$ acts trivially on $R$.

We now define a twisted version of Khovanov homology. Let $p_{1}, \ldots, p_{n}$ be a collection of markings on the edges of a link diagram and $w_{i} \in R$ be weights associated to those markings. In addition to crossings, we add another type of elementary diagram: an edge with a single marking of weight $w \in R$, which we depict as $+w$ (and often just as + if the weight is clear from context). We define $K h(+w):=\mid$ with differential $w$. The reduced twisted Khovanov homology associated to a given marking can now be defined by taking the tensor product corresponding to gluing the crossings and markings. See Figure 1 for an example.

Notice that the differential of the crossings has homological degree 2 and $q$-degree 0 , whereas the differential of markings has homological degree 0 


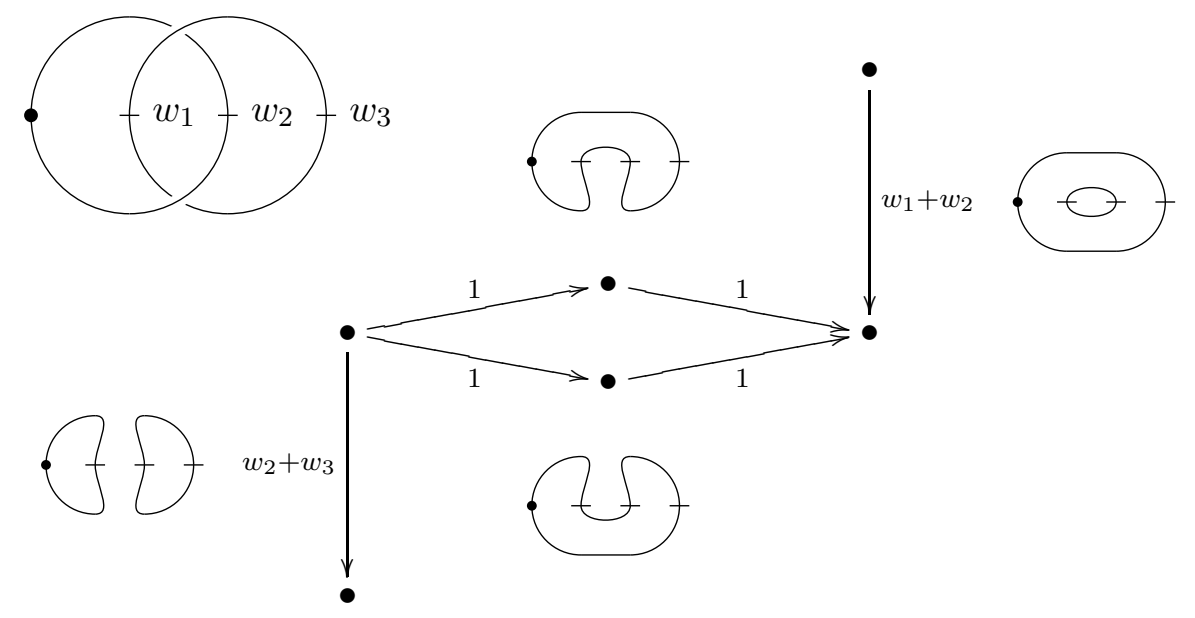

Figure 1. Twisted Khovanov homology of the Hopf link. Basepoint and weights are as indicated at the top left of the figure. The rest of the figure shows the differential of the complex, where each dot represents a copy of $R$. The complex can still be viewed as a cube of resolutions; the four resolutions of the link are drawn near their corresponding subquotient complexes. Notice that the horizontal differential is identical to the differential of the reduced Khovanov homology of the Hopf link.

and $q$-degree -2 . In order for the definition to make sense, we therefore need to collapse the two gradings to a single $\delta$-grading, where (with the conventions used above) the $\delta$-grading is the difference of homological and $q$-grading (note that our homological grading is twice the grading one usual considers in Khovanov homology).

\section{Properties of twisted Khovanov homology}

In this section we prove the following invariance result.

Theorem 3.1. Let $D$ be the diagram of a tangle with markings of weight $w_{i 1}, \ldots, w_{i n_{i}}$ on the ith component of the tangle. Then the isomorphism type $K h(D)$ depends only on $w_{i}:=\sum_{j=1}^{n_{i}} w_{i j}$.

Corollary 3.2. If $K$ is a knot then $\overline{K h}(K)$ does not depend on the markings, in particular, it is isomorphic to $\delta$-graded Khovanov homology.

Proof. By the theorem above (technically applied to the knot cut open at the basepoint), we may move all markings to the same edge that the basepoint lies on. Thus the differential of $K h(K)$ is $d+w x$, where $d$ is the differential of the untwisted Khovanov homology, $w$ is the sum of all the weights, and $x$ is the action of the dot at the basepoint as before. Hence the differential on $\overline{K h}(K)$ is simply $d \otimes 1$. 
of Theorem 3.1. First, note that the Khovanov complex of two adjacent markings of weight $w_{1}$ and $w_{2}$ is $K h(\ddagger)=\mid$ with differential $d=w_{1} \downarrow \otimes 1+$ $1 \otimes w_{2} \phi=\left(w_{1}+w_{2}\right) \dagger$.

Next, we prove that we can slide a marking past a crossing:

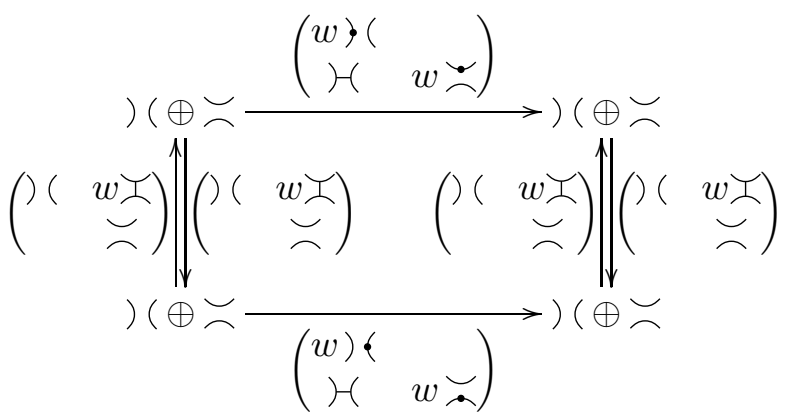

The top row is the differential of $K h\left(X^{\prime}\right)$ and the bottom row is the differential of $K h\left(\searrow_{X}\right)$. The arrows pointing up and down define an isomorphism between $K h\left(\chi^{\prime}\right)$ and $K h\left(\searrow^{\prime}\right)$, as can be easily checked. An isomorphism between $K h\left(\lambda^{\times}\right)$and $K h\left(\searrow^{\searrow}\right)$ can be established in a similar fashion.

Finally, we note that since we can always slide markings away from tangles without closed components, invariance of the homotopy type of $K h(L)$ under Reidemeister moves follows immediately from the invariance of the untwisted theory.

\section{Spanning TRees}

The version of twisted Khovanov homology defined in this note admits a spanning tree model along the same lines as the models in [1] and [6]. $\overline{K h}(L)$ has a natural double complex structure: The horizontal differential is simply the differential of untwisted reduced Khovanov homology, whereas the vertical differential is induced by the weights. This double complex structure gives rise to a spectral sequence converging to $\overline{K h}(L)$.

If $R$ is a field and each edge of the graph (with the exception of the edge that contains the basepoint) has a non-zero weight associated to it and all weights are linearly independent over $\mathbb{F}_{2}$, we can describe the spectral sequence explicitly: The $d_{0}$ differential cancels all states corresponding to disconnected resolutions of the diagram and the remaining states are in one-to-one correspondence with spanning trees. For grading reasons, all subsequent differentials except for the $d_{2}$ differential are zero and an explicit calculation shows that the $d_{2}$ differential between two resolutions $R$ and $R^{\prime}$ that differ at two places (such that $R$ is the 0-resolution at those places and $R^{\prime}$ is the 1-resolution) is $\frac{1}{\sum_{i} w_{i}}+\frac{1}{\sum_{i} w_{i}^{\prime}}$, where $w_{i}$ and $w_{i}^{\prime}$ are the weights on the circles not containing the basepoint in the two intermediate resolutions between $R$ and $R^{\prime}$. Otherwise, $d_{2}=0$. 


\section{Relation to Roberts' totally twisted Khovanov homology}

In this section we show that the theory described earlier coincides with Roberts' totally twisted Khovanov homology. Robert's theory is defined over the ring $\mathbb{F}_{2}\left(x_{1}, \ldots, x_{n}\right)$, where the $x_{i}$ are labels associated to the regions of the knot diagram. The variables associated to the two regions $x_{n-1}$ and $x_{n}$ adjacent to the basepoint are never used in the differential, hence we may consider the same theory over the ring $R^{\prime}:=\mathbb{F}_{2}\left(x_{1}, \ldots, x_{n-2}\right)$. Define vector spaces $X:=\mathbb{F}_{2}\left\langle x_{1}, \ldots, x_{n-2}\right\rangle$ and $Y:=\mathbb{F}_{2}\left\langle y_{1}, \ldots, y_{m}\right\rangle$, where the $y_{j}$ are variables associated to the edges of the diagram not containing the basepoint and define a linear map $f: X \rightarrow Y$ by sending $f\left(x_{i}\right)$ to the sum of the $y_{j}$ associated to the edges that make up the boundary of the region labeled $x_{i}$. We claim that $f$ is injective. Assume that $f\left(\sum_{i} \lambda_{i} x_{i}\right)=0$. If $x_{i_{1}}$ and $x_{i_{2}}\left(i_{1}, i_{2} \leq n-2\right)$ are the labels of two adjacent regions, then $\lambda_{i_{1}}=\lambda_{i_{2}}$ since the $y_{j}$ associated to their common edge does not appear in any of the $f\left(x_{i}\right)$ for $x \neq x_{i_{1}}, x_{i_{2}}$. Thus $\lambda_{1}=\ldots=\lambda_{n-2}$. By considering an edge adjacent to $x_{n-1}$ or $x_{n}$, we see that in fact $\lambda_{i}=0$ for all $1 \leq i \leq n-2$, hence $f$ is injective as claimed. Fix a linear map $g: Y \rightarrow X$ satisfying $g \circ f=\operatorname{id}_{X}$. Note that if $C$ is a basepoint-avoiding circle in any resolution of the diagram, then the sum over all $x_{i}$ corresponding to the region of $S^{2} \backslash C$ not containing the basepoint is the same as the sum over $g\left(y_{j}\right)$, where $y_{j}$ runs over the edges of $C$. Thus we can obtain Robert's theory by working over the ring $R^{\prime}$ and assigning the weight $w_{j}:=g\left(y_{j}\right)$ to a marking on the $j$ th edge.

Similarly, given weights $w_{j} \in R$, we obtain the theory described in this note from Roberts' definitions by specializing $x_{i}$ to be the sum of the weights of the markings on the boundary of the region labeled $x_{i}$.

\section{REFERENCES}

[1] John Baldwin and Adam Levine, A combinatorial spanning tree model for knot Floer homology, 2011. arXiv:1105.5199v2 [math.GT].

[2] Dror Bar-Natan, Khovanov's homology for tangles and cobordisms, Geom. Topol. 9 (2005), 1443-1499 (electronic).

[3] , Fast Khovanov homology computations, J. Knot Theory Ramifications 16 (2007), no. 3, 243-255.

[4] Abhijit Champanerkar and Ilya Kofman, On mutation and Khovanov homology, Commun. Contemp. Math. 10 (2008), no. suppl. 1, 973-992.

[5] Peter Ozsváth and Zoltán Szabó, Heegaard Floer homology and alternating knots, Geom. Topol. 7 (2003), 225-254 (electronic).

[6] Lawrence Roberts, Totally Twisted Khovanov Homology, 2011. arXiv:1109.0508v1 [math.GT].

[7] S. Wehrli, A spanning tree model for Khovanov homology, J. Knot Theory Ramifications 17 (2008), no. 12, 1561-1574.

E-mail address: tcjaeger@syr.edu

Department of Mathematics; 215 Carnegie Building; Syracuse University; SYRACUSE, NY 13244-1150 